\title{
Grundlagen und derzeitige Entwicklungen der Messunsicherheitsbewertung - Von der Fehlerrechnung zur Bayes-basierten Messdatenauswertung
}

\author{
Klaus-Dieter Sommer \\ Physikalisch-Technische Bundesanstalt (PTB), Braunschweig, Germany \\ Raghu Kacker \\ National Institute of Standards and Technology (NIST), Gaithersburg, USA
}

\begin{abstract}
Classical error analysis is increasingly replaced with uncertainty evaluation in accordance with the ISO-GUM which in fact nowadays is a de facto standard for evaluating uncertainty in measurement.

Classical error analysis considers the measurement error as relevant carrier of information about the real behaviour of a measuring process, a sub-process or an individual effect of an influence/disturbance. Error analysis overemphasises the statistical analysis of observed data, treats non-statistical information unsatisfactorily and cannot take account of logically correlated input quantities properly. The mathematical basis for error analysis is formed by statistical sampling analysis.
\end{abstract}

The ISO-GUM propagates estimates and uncertainties through a linear approximation of the measurement equation. In this sense, the ISO-GUM is a robust general purpose approach. It requires the modelling of the interrelation of the input quantities with the measurand(s) and it represents non-statistical knowledge about quantities and parameters in a Bayesian way by appropriate probability density functions. It might be concluded that the ISO-GUM provides a degree-of-belief interpretation of classical formulas. Its application limits are given with significantly non-linear systems and, regarding the output quantity, violation of the central limit theorem.

A draft supplement to the ISO-GUM propagates probability density functions through numerical simulation. The mathematical basis for this method is the Bayesian probability theory.

The paper describes the development from error analysis to Bayesian measurement data evaluation and demonstrates the need and consistency of the ongoing advancements in measurement data analysis and evaluation of uncertainties. A generalized measuring process is employed to illustrate and point out the basic GUM concepts and its practical procedures as well as to discuss the merits and limitations of classical error analysis. Modelling is interpreted as a Bayesian learning process. Emphasis is put to non-statistical evaluation of information, denoted as Typ-B by the ISO-GUM, and problems hidden in analising repeated measurements, denoted as Type-A, are explicated briefly, too. The paper shows, that the GUM approach is consistent with the Bayesian view of pdfs as representing the state of knowledge based on the principle of maximum information entropy and Bayes' theorem.

The paper concludes that Bayesian concepts provide consistent and powerful tools for uncertainty evaluation. 


\section{Autoren:}

Dir. und Prof. Dr.-Ing. Klaus-Dieter Sommer, Physikalisch-Technische Bundesanstalt, Abteilung Chemische Physik und Explosionsschutz, Bundesallee 100, 38116 Braunschweig, Tel.-Nr.: 0531592 3010, Fax-Nr.: 0531592 3015,

Email: klaus-dieter.sommer@ptb.de

Raghu N. Kacker, National Institute of Standards and Technology (NIST), $100 \mathrm{Bu}$ reau Drive, Stop 8910, Gaithersburg, MD 20899-8910, Email: raghu.kacker@nist.gov

Literatur:

Kacker R., Sommer K.-D., Kessel R.

Evolution of modern approaches to express uncertainty in measurement Metrologia, 2007, 44, ${ }^{\circ} 6,513-529$ 